\title{
Transgenic amplification of glucocorticoid action in adipose tissue causes high blood pressure in mice
}

\author{
Hiroaki Masuzaki, ${ }^{1}$ Hiroshi Yamamoto, ${ }^{1}$ Christopher J. Kenyon, ${ }^{2,3}$ Joel K. Elmquist, ${ }^{1,4}$ \\ Nicholas M. Morton, ${ }^{2}$ Janice M. Paterson, ${ }^{2,3}$ Hiroshi Shinyama, ${ }^{1}$ Matthew G.F. Sharp, ${ }^{3}$ \\ Stewart Fleming, ${ }^{5}$ John J. Mullins, ${ }^{3}$ Jonathan R. Seckl, ${ }^{2}$ and Jeffrey S. Flier ${ }^{1}$

\begin{abstract}
${ }^{1}$ Division of Endocrinology, Diabetes, and Metabolism, Department of Medicine, Beth Israel Deaconess Medical Center and Harvard Medical School, Boston, Massachusetts, USA

${ }^{2}$ Endocrinology Unit, Molecular Medicine Center, and

${ }^{3}$ Molecular Physiology Laboratory, University of Edinburgh, Edinburgh, United Kingdom Boston, Massachusetts, USA

${ }^{5}$ Department of Cellular and Molecular Pathology, University of Dundee and Ninewells Hospital, Dundee, United Kingdom
\end{abstract} \\ ${ }^{4}$ Department of Neurology and Program in Neuroscience, Beth Israel Deaconess Medical Center and Harvard Medical School,
}

\begin{abstract}
Obesity is closely associated with the metabolic syndrome, a combination of disorders including insulin resistance, diabetes, dyslipidemia, and hypertension. A role for local glucocorticoid reamplification in obesity and the metabolic syndrome has been suggested. The enzyme $11 \beta$-hydroxysteroid dehydrogenase type 1 (11ß-HSD1) regenerates active cortisol from inactive 11-keto forms, and aP2-HSD1 mice with relative transgenic overexpression of this enzyme in fat cells develop visceral obesity with insulin resistance and dyslipidemia. Here we report that aP2-HSD1 mice also have high arterial blood pressure (BP). The mice have increased sensitivity to dietary salt and increased plasma levels of angiotensinogen, angiotensin II, and aldosterone. This hypertension is abolished by selective angiotensin II receptor AT-1 antagonist at a low dose that does not affect BP in non-Tg littermates. These findings suggest that activation of the circulating renin-angiotensin system (RAS) develops in aP2-HSD1 mice. The longterm hypertension is further reflected by an appreciable hypertrophy and hyperplasia of the distal tubule epithelium of the nephron, resembling salt-sensitive or angiotensin II-mediated hypertension. Taken together, our findings suggest that overexpression of 11 $\beta$-HSD1 in fat is sufficient to cause saltsensitive hypertension mediated by an activated RAS. The potential role of adipose $11 \beta-H S D 1$ in mediating critical features of the metabolic syndrome extends beyond obesity and metabolic complications to include the most central cardiovascular feature of this disorder.
\end{abstract}

J. Clin. Invest. 112:83-90 (2003). doi:10.1172/JCI200317845.

\section{Introduction}

Obesity is often associated with the metabolic syndrome, which includes insulin resistance, dyslipidemia, and hypertension (1-3). Weight reduction lowers arterial blood pressure (BP) in obese hypertensive patients, suggesting a close association between energy homeostasis and $\mathrm{BP}(2,4,5)$. The mechanisms underlying hypertension in obesity, however, have not been fully elucidated.

Received for publication January 14, 2003, and accepted in revised form April 22, 2003.

Address correspondence to: Jeffrey S. Flier, Division of Endocrinology, Diabetes, and Metabolism, Department of Medicine, Beth Israel Deaconess Medical Center and Harvard Medical School, E/Finard 202, 300 Brookline Avenue, Boston, Massachusetts 02215, USA. Phone: (617) 667-9050;

Fax: (617) 667-9054; E-mail: jflier@caregroup.harvard.edu. Hiroshi Yamamoto and Christopher J. Kenyon contributed equally to this work.

Conflict of interest: The authors have declared that no conflict of interest exists.

Nonstandard abbreviations used: arterial blood pressure (BP); angiotensinogen (AGT); renin-angiotensin system (RAS); transgenic (Tg); $11 \beta$-hydroxysteroid dehydrogenase type 1

(11ß-HSD1); adipocyte fatty acid binding protein (aP2); heart rate (HR); mean arterial pressure (MAP); angiotensin II receptor AT-1 (AT-1 receptor); radioimmunoassay (RIA).
Hyperinsulinemia, frequently associated with obesity and insulin resistance, also causes excessive insulin action in a tissue-specific manner in kidney, arterial walls, and sympathetic nervous system (1-3). This results in BP elevation through sodium retention, proliferation of VSMCs, and sympathetic activation, respectively $(3,4,6)$. Furthermore, insulin resistance occurring in vascular endothelial cells may reduce nitric oxide synthesis, which leads to arterial constriction and $\mathrm{BP}$ elevation $(7,8)$.

A line of evidence has suggested that adipocytederived hypertensive substances such as leptin and angiotensinogen (AGT) are also involved in the pathogenesis of obesity-related hypertension $(2-4,9)$. Leptin, secreted in excess from enlarged adipose tissue, activates sympathetic nervous system pathways including those regulating BP (2-4). The renin-angiotensin system (RAS) has also been firmly established as a major determinant of $\mathrm{BP}(10,11)$. AGT, secreted mainly from liver and adipose tissue, serves as the unique substrate of renin and initiates RAS activation $(12,13)$. Epidemiological studies have reported a significant correlation between plasma AGT concentrations and BP or BMI in some human populations $(14,15)$. A recent 
study by Massiera et al. has demonstrated that overexpression of AGT exclusively in adipose tissue in transgenic (Tg) mice results in significant elevation of BP, suggesting a possible pathophysiologic role of adipose AGT in obesity-related hypertension (16).

Glucocorticoids play a pivotal role in regulating fat metabolism, function, and distribution (3, 9, 17). Patients with Cushing syndrome, a rare disease of systemic glucocorticoid excess caused by pituitary or adrenal tumors, develop reversible visceral obesity with insulin resistance, dyslipidemia, and hypertension (17-21). However, the pathophysiologic role of glucocorticoids in the prevalent forms of obesity and in the metabolic syndrome has remained obscure because circulating cortisol levels are not elevated in the majority of obese subjects $(22,23)$.

Recently, locally enhanced activity of glucocorticoids in tissues such as adipose tissue and skeletal muscle has been demonstrated in the metabolic syndrome (24-28). Activity of $11 \beta$-hydroxysteroid dehydrogenase type 1 $(11 \beta$-HSD1) is significantly increased in fat tissue from obese humans and rodents, suggesting a role for local glucocorticoid reamplification in obesity and the metabolic syndrome $(25,26,28-30)$. In accordance with this notion, $11 \beta$-HSD1 knockout mice exhibit an apparent insulin-sensitive phenotype $(31,32)$ and resist visceral fat accumulation even when fed a high-fat diet (33). On the other hand, Tg mice overexpressing $11 \beta$-HSD1 in fat tissue (aP2-HSD1 mice) show an increase in enzyme activity to an extent similar to that seen in obese humans, and replicate visceral fat accumulation with insulin and leptin resistance and dyslipidemia (34). Thus, the aP2-HSD1 mouse is a unique model for studying the pathophysiology of the human metabolic syndrome.

A major unanswered question is whether aP2-HSD1 mice also develop hypertension, a critical feature of the metabolic syndrome. Here we show that Tg mice overexpressing 11 $\beta$-HSD1 in adipose tissue have hypertension accompanied by activation of the systemic RAS and salt sensitivity.

\section{Methods}

Animals. Generation of Tg mice overexpressing $11 \beta$-HSD1 under the control of the enhancer-promoter region of the adipocyte fatty acid-binding protein (aP2) gene (aP2-HSD1 mice) has been reported elsewhere (34). In these mice, transgene-derived mRNA for rat $11 \beta$-HSD1 was expressed specifically in adipose tissue, and due to $\mathrm{Tg}$ regeneration of active glucocorticoid from inactive forms in adipose tissue, concentrations of corticosterone (an active form of glucocorticoid in rodents) in fat depots was significantly increased. Mice were FVB congenic strain crosses, WT or hemizygous for the transgene. Unless otherwise indicated, mice were fed a chow diet (1\% salt; Harlan Teklad Laboratory, Madison, Wisconsin, USA) and kept on a cycle of 10 hours of light (0700-1700 hours) and 14 hours of darkness. Male aP2-HSD1 mice, 19-27 weeks old, from line 10 (34) and non- $\mathrm{Tg}$ littermates were used in the present study. The
Animal Care and Use Committee at Beth Israel Deaconess Medical Center and Harvard Medical School approved all studies.

Surgical procedures and measurement of blood pressure. Mice (23-26 weeks of age) were anesthetized with isoflurane (5\% for induction, $2 \%$ for maintenance) and were implanted in the left carotid artery with a catheter coupled with a sensor and transmitter (TA11PA-C20; Data Sciences International., St. Paul, Minnesota, USA) $(35,36)$. Telemetry signals were received by an antenna below the cage that relayed the signals to a signal processor (DataQuest ART Gold version 2.1; Data Sciences International) connected to a Compaq desktop PC (Compaq Computer Corp., Houston, Texas, USA). These transmitters allow remote monitoring of BP and heart rate $(\mathrm{HR})$ in unanesthetized, unrestrained mice. BP and HR were monitored every minute for 9 days beginning 1 week after the surgery. Results are expressed as mean \pm SEM of 60 values each hour.

High-salt diet experiments. The aP2-HSD1 mice and non-Tg littermates 19 weeks of age were given a highsalt diet containing $8 \%$ salt $(8 \% \mathrm{NaCl}$ Diet; Harlan Teklad Laboratory) for 3 weeks, and BP and HR were monitored during the course of the experiments.

Pharmacologic blockade of the RAS. To assess the activation of the RAS in mice, under continuous telemetry monitoring of mean arterial pressure (MAP) and HR, the specific angiotensin II receptor AT-1 (AT-1 receptor) antagonist GA0113 (37) (Mitsubishi Pharma Corp., Yokohama, Japan) was administered orally once a day (at 1500 hours) for 4 days, and the effect of drug administration was evaluated at day 5. On examination of the dose-related effects of GA0113, we found that the maximal dose that does not lower MAP in non-Tg mice is $0.1 \mathrm{mg} / \mathrm{kg}$ body weight. The long plasma terminal half-life (12.2 hours) and excellent oral bioavailability (94\%) of this compound enabled us to give the drug once a day to mice (37). A previous study using renal artery-ligated hypertensive rats indicated that repeated administration of GA0113 at this dose showed gradually potentiated reduction of elevated BP that reached a plateau after 4 days of treatment without alteration of HR (37).

Intraperitoneal insulin tolerance testing. After a 6-hour fast, mice were injected intraperitoneally with 0.75 $\mathrm{U} / \mathrm{kg}$ body weight insulin (Novolin R; Novo Nordisk A/S, Bagsvaerd, Denmark) (34). Blood was sampled from the tail vein before and 15,30,60, and 90 minutes after the injection and blood glucose levels were determined using a glucometer (One Touch II; LifeScan Inc., Milpitas, California, USA).

Hormone assays and $m R N A$ expression. Plasma AGT levels were measured by radioimmunoassay (RIA) as equivalent amounts of angiotensin I (38). Plasma angiotensin II levels were measured by HPLC coupled with RIA (38). Plasma aldosterone levels were also measured using an RIA kit (Diagnostic Products Corp., Los Angeles, California, USA). Plasma renin concentrations were measured as reported (39). AGT mRNA levels in mesenteric 
adipose depots and liver, and AT-1 receptor mRNA levels in kidney and abdominal aorta, were determined by quantitative ${ }^{32} \mathrm{P}$ RT-PCR with these primers: AGT (34) sense ( $5^{\prime}$-CCTGAAGGCCACCATCTTCT- $\left.3^{\prime}\right)$ and antisense ( $5^{\prime}$-GATCATTGCGACCTGGGCAG-3') and AT-1 receptor (40) sense ( $5^{\prime}$-CACCTATGTAGATCGCTTC- $\left.3^{\prime}\right)$ and antisense $\left(5^{\prime}\right.$-GCACAATCGCCATAATTATCC- 3 '). The results were normalized to the signal generated for $\beta$-actin and cyclophilin mRNA's (Ambion Inc., Austin, Texas, USA).

Histological preparation and in situ bybridization. Kidneys and adrenal glands were fixed in $10 \%$ buffered formalin and embedded in paraffin wax, and multiple adjacent $4-\mu \mathrm{m}$ sections were cut and mounted on glass slides. After dehydration, the sections were stained with $H \& E$. Sections were examined by an experienced pathologist (S. Fleming) who was blinded to the genotype. Using adrenal gland tissue, in situ hybridization for aldosterone synthetase and 11ß-hydroxylase mRNA's (41) was performed using antisense ${ }^{35}$ S-labeled cRNA probes. Control procedures using sense probes were performed to confirm specificity. Images of five or six sections of each gland were quantified autoradiographically and then statistically evaluated.

Electron microscopy. Kidney tissue for electron microscopy was immersion-fixed as 1 -mm cubes in $4 \%$ glutaraldehyde in carbohydrate buffer. The tissue blocks were postfixed in osmium tetroxide, washed in carbohydrate buffer, and dehydrated through a graded alcohol series. Blocks were embedded in araldite resin. Ultra-thin sections were cut, mounted on copper grids, and stained with uranyl acetate and lead citrate. They were examined on a Phillips CM-12 transmission electron microscope as previously described (39).

Statistical analyses. Data are expressed as mean \pm SEM. Differences were assessed by unpaired two-tailed $t$ test or ANOVA where applicable. Differences in MAP and HR at the different timepoints between two groups (non- $\mathrm{Tg}$ vs. $\mathrm{Tg}$ mice or untreated vs. drug-treated mice) were analyzed by repeated measures ANOVA followed by the Student $t$ test. (StatView 4.01; Abacus Concepts Inc., Berkeley, California, USA).

\section{Results}

Elevated MAP in aP2-HSD1 mice. As expected (34), at 24 weeks of age, body weight of aP2-HSD1 mice (Tg mice) was mildly but significantly increased relative to nonTg littermates $(35.1 \pm 2.1 \mathrm{~g}, n=7$, vs. $30.4 \pm 1.6 \mathrm{~g}, n=5$, respectively; $P<0.03$ ). When fed ad libitum, blood glucose, plasma insulin, and plasma leptin levels in $\mathrm{Tg}$ mice were significantly elevated (glucose, $172 \pm 16$ vs. $124 \pm 9 \mathrm{mg} / \mathrm{dl}, P<0.02$; plasma insulin: $792 \pm 105$ vs. $232 \pm 42 \mathrm{pg} / \mathrm{ml}, P<0.01$; leptin: $6,912 \pm 1,012$ vs. $2,190 \pm 326 \mathrm{pg} / \mathrm{ml}, P<0.01$, respectively), indicating the presence of insulin and leptin resistance in $\mathrm{Tg}$ mice.

To explore the cardiovascular consequences of $\mathrm{Tg}$ amplification of glucocorticoid action in fat tissue, BP and HR were assessed by telemetry. MAP in both non$\operatorname{Tg}(n=14)$ and $\operatorname{Tg}(n=17)$ mice at 23 weeks of age showed a monophasic circadian variation (42) with a nadir at around 1100-1400 hours and a peak at around 2000-2300 hours (Figure 1a). Throughout the day, MAP in Tg mice was significantly elevated (by 10-30 $\mathrm{mmHg})$ compared with non-Tg mice $(P<0.04)$. Notably, a remarkable rise during the early dark phase was observed, indicating that MAP elevation in Tg mice is exaggerated in the active phase. This is reminiscent of a morning surge that is often observed in human essential hypertension with multiple metabolic risks $(43,44)$. No significant difference in HR was noted between non$\mathrm{Tg}$ and $\mathrm{Tg}$ mice throughout the day (data not shown).

There are several possible mechanisms for the hypertension associated with the metabolic syndrome. Evidence has indicated that even in the presence of insulin and leptin resistance, compensatory hyperinsulinemia and hyperleptinemia play pivotal roles in the pathogenesis of obesity-related hypertension $(4,6,45)$. Thus, relative hyperinsulinemia and hyperleptinemia in aP2-HSD1 mice are likely to contribute, at least in part, to BP elevation.

Circulating renin-angiotensin-aldosterone system is activated in aP2-HSD1 mice. AGT functions as a unique substrate of renin that is secreted mainly from liver and adipose tissue (10-12). AGT mRNA in adipocytes is regulated by nutritional manipulation and upregulated by glucocorticoids $(46,47)$. Notably, the AGT mRNA level is significantly higher in visceral fat than in subcutaneous fat and is elevated in fat depots from obese humans $(14,15,48$, 49), suggesting that adipose AGT potentially links the metabolic syndrome with hypertension.

The mRNA expression of AGT in mesenteric fat from $\mathrm{Tg}$ mice was significantly increased compared with non-Tg mice $(372 \pm 21, n=5$ vs. $100 \pm 2, n=5$, respectively, in arbitrary units). The liver AGT mRNA level tended to increase in $\mathrm{Tg}$ mice, but the difference was not statistically significant. A comparison of AGT mRNA levels in mesenteric fat of $\mathrm{Tg}$ mice and non- $\mathrm{Tg}$ mice yielded values (in arbitrary units) of $472 \pm 49$ and $412 \pm 28$, respectively. Collectively, the plasma AGT level in Tg mice was also significantly elevated $(201 \pm 14$ nmol/l, $n=7$ vs. $159 \pm 10, n=9$, respectively; $P<0.03$ ), further supporting a role for adipose AGT and local glucocorticoid in the regulation of plasma AGT levels.

These results prompted us to hypothesize that the activation of the RAS may also be involved in the development of high BP in Tg mice. Consistent with this notion, plasma levels of angiotensin II (Tg: $91.8 \pm 5.2$ $\mathrm{pg} / \mathrm{ml}, n=6$ vs. non-Tg: $19.8 \pm 0.8 \mathrm{pg} / \mathrm{ml}, n=3$; $P<0.001)$ and aldosterone (Tg: $1,117 \pm 142 \mathrm{pmol} / \mathrm{l}, n=5$ vs. non-Tg: $510 \pm 78, n=5 ; P<0.03$ ) were significantly elevated in $\mathrm{Tg}$ mice compared with non-Tg mice, indicating that "substrate excess" can potentiate components of the RAS in mice. No appreciable difference between the two groups was observed in AT-1 receptor mRNA expression level in kidneys or aortas (data not shown). It is interesting to note that plasma renin concentrations in $\mathrm{Tg}$ mice were also increased $(1,894 \pm 413$ $\mathrm{ng} / \mathrm{ml} / \mathrm{h}, n=5)$ versus non- $\mathrm{Tg}$ mice $(886 \pm 136, n=5$; $P<0.03$ ), suggesting impaired negative feedback on circulating RAS by angiotensin II and/or stimulation of 
renin secretion (e.g., by insulin resistance and sympathetic activation) $(2,3,11,45)$. It is unclear at present whether such observations in $\mathrm{Tg}$ mice are consistent with human pathophysiology. It has been shown that renin secretion is increased by renal sympathetic activation $(10,12,40)$, and that both hyperinsulinemia and hyperleptinemia enhance sympathetic nerve activity in a tissue-specific manner (e.g., kidney, central nervous system) (2-4). Thus, it is tempting to speculate that in $\mathrm{Tg}$ mice, hyperinsulinemia and hyperleptinemia activate the sympathetic nervous system and cause subsequent renin secretion. Further studies are required to clarify whether such a mechanism is operative in human hypertension in patients with the metabolic syndrome.

Adrenal 11ß-hydroxylase and aldosterone synthetase are closely related isozymes with distinct roles in glucocorticoid and mineralocorticoid production in adrenal cortex (41). 11ß-hydroxylase is expressed at higher levels in the zona fasciculata, catalyzing the final reaction in the production of corticosterone. Aldosterone synthetase catalyzes the final step in aldosterone biosynthesis, which is expressed only in the zona glomerulosa $(10,11)$. In situ hybridization analyses of adrenal gland from $\mathrm{Tg}$ mice $(n=3)$ revealed a significant increase in aldosterone synthetase mRNA (increase of $31 \%, P<0.02)$ compared with that in non-Tg mice $(n=5)$, while $11 \beta$-hydroxylase mRNA of $\mathrm{Tg}$ mice was decreased (decrease of $38 \%$, $P<0.02$ ) (Figure 1b). These results confirm that the circulating RAS was activated in $\mathrm{Tg}$ mice. These data also suggest that in response to the adipose overproduction of glucocorticoid, negative feedback of the hypothalamic-pituitary-adrenal axis suppressed adrenal glucocorticoid production to maintain the documented normal circulating glucocorticoid levels in Tg mice (34).

To further evaluate the involvement of RAS activation in high BP in Tg mice, the specific AT-1 receptor antagonist GA0113 was administered with continuous telemetry monitoring of MAP and HR. The MAP profile at day 5 of treatment demonstrated that elevated $\mathrm{BP}$ in $\mathrm{Tg}$ mice $(n=6,27$ weeks of age) was markedly abrogated by administra- tion of GA0113 at a low dose $(0.1 \mathrm{mg} / \mathrm{kg}$ body weight). This dose did not decrease MAP in non-Tg littermates $(n=5)$ (Figure 1c). As the pressure-lowering effect was exaggerated during dark phase (about $15-30 \mathrm{mmHg}$ decrease) in Tg mice, the daily MAP profile changed into a flatter curve without a distinct nadir (Figure 1c). Taken together, these results indicate that activated RAS contributes considerably to high BP in Tg mice. During the course of drug administration, HR changes were not observed in either group (data not shown). Telemetry monitoring was continued for 4 days after the final administration. We observed that MAP returned to the initial values (data not shown), indicating that the drug intervention did not cause irreversible tissue damage.

Hypertension in aP2-HSD1 mice is salt-sensitive. Salt sensitivity is a common trait in patients with essential hypertension, with a prevalence estimated at greater than $50 \%(50,51)$. Although many factors influence the $\mathrm{BP}$ response to a high-salt diet, the RAS has been demonstrated to play a central role in this process (50-52). Compared with salt-resistant subjects, salt-sensitive individuals exhibit a blunted RAS response when
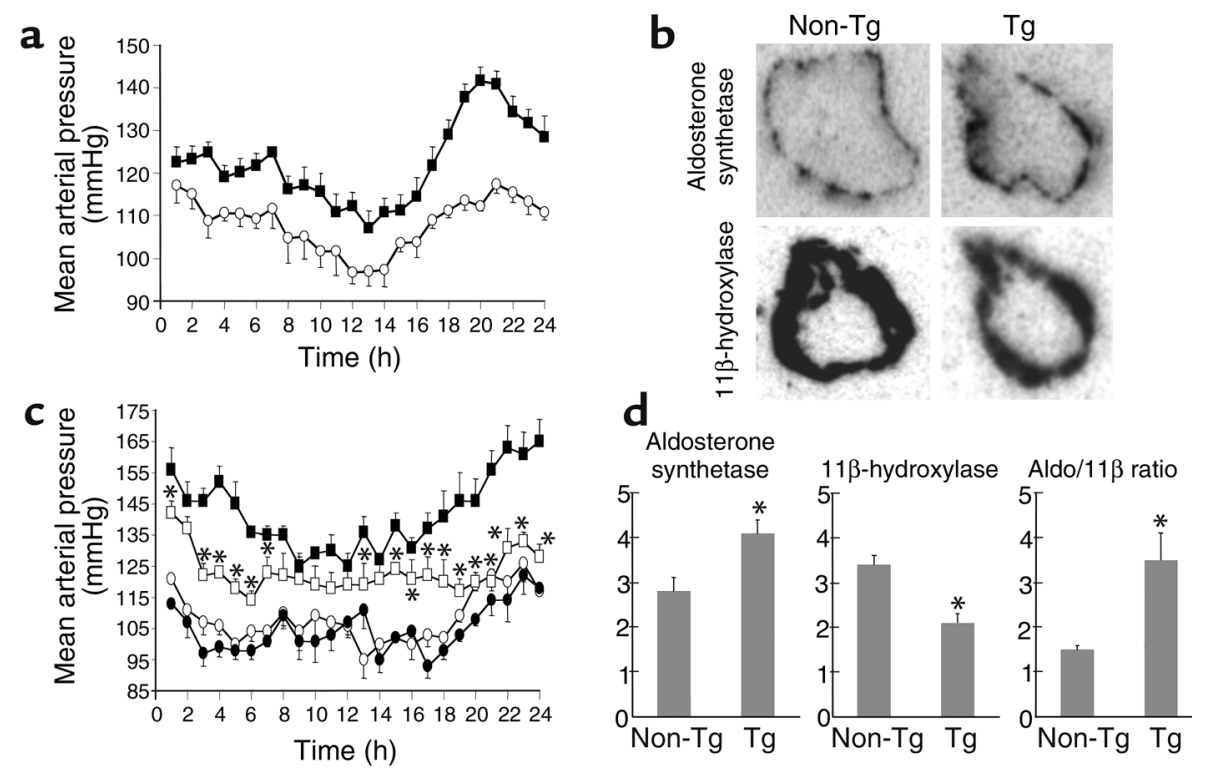

Figure 1

(a) Daily profile of MAP in aP2-HSD1 mice at 23 weeks of age. MAP was monitored closely by telemetry transmitters implanted in the left carotid artery. Values are expressed as mean \pm SEM of 60 data points each hour. Throughout the day, MAP in Tg mice ( $n=17$, filled squares) was significantly elevated (by 10-30 mmHg, $P<0.04$ ) compared with that of non-Tg mice ( $n=14$, open circles). (b) In situ hybridization analysis of adrenal glands from aP2-HSD1 mice. Adrenal gland tissue from non- $\mathrm{Tg}$ $(n=5)$ and Tg mice $(n=3)$ was hybridized with ${ }^{35}$ S-labeled cRNA probes for aldosterone synthetase and $11 \beta$-hydroxylase. Images of five or six sections from each gland were quantified and statistically evaluated (d). Results are expressed as arbitrary units. The ratio of aldosterone synthetase to $11 \beta$-hydroxylase (Aldo/11 $)$ ) in each sample was also determined. ${ }^{*} P<0.02$ versus non-Tg. (c) Effect of specific AT-1 receptor antagonist GA0113 on MAP in aP2-HSD1 mice at 27 weeks of age. GA0113 was administered orally once per day $(0.1 \mathrm{mg} / \mathrm{kg}$ body weight, at 1500 hours $)$ for 4 days, and the effect of drug administration was evaluated by telemetry on day 5 . Filled circles, non- $\operatorname{Tg}(n=5)$ initial (untreated) values; open circles, non- $\operatorname{Tg}$ treated; filled squares, $\operatorname{Tg}(n=6)$ initial (untreated); open squares, $\mathrm{Tg}$ treated. ${ }^{*} P<0.05 \mathrm{vs}$. Tg untreated. Telemetry monitoring was continued for 4 days after the final administration to observe that MAP in both sets of mice returned to initial values. 
a

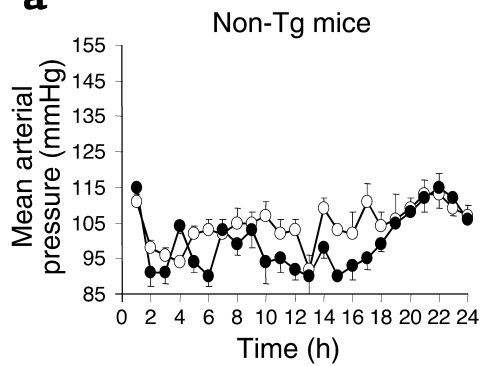

b

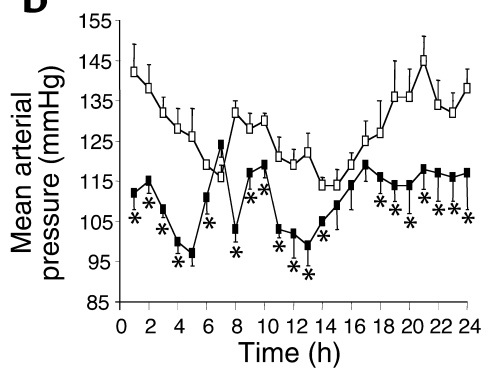

Tg mice

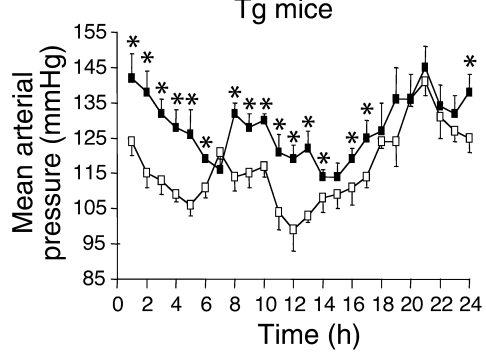

Figure 2

(a) Effect of high-salt diet on MAP in aP2-HSD1 mice. The aP2-HSD1 mice and non-Tg littermates at 19 weeks of age were given a high-salt (8\%) diet for 3 weeks. The graph on the left shows that MAP in non- $\operatorname{Tg}$ mice $(n=4)$ fed a high-salt diet (filled circles) did not elevate significantly compared with the initial values when fed a chow diet with $1 \%$ salt (open circles). The graph at right shows that MAP in Tg mice fed a high-salt diet (filled squares) $(n=5)$ was significantly elevated during most of the day (except 0700 hours to 0800 hours, 1500 hours to 1600 hours, and 1800 hours to 2400 hours), by $10-20 \mathrm{mmHg}$ compared with $\mathrm{Tg}$ mice fed a normal diet (open squares). ${ }^{*} P<0.05$ vs. Tg mice fed a chow diet. (b) Effect of the specific AT-1 receptor antagonist GA0113 on MAP in aP2-HSD1 mice fed a high-salt diet. Four days of low-dose administration of GA0113 $(0.1 \mathrm{mg} / \mathrm{kg}$ body weight, which does not lower MAP in non-Tg mice) to $\mathrm{Tg}$ mice fed a high-salt diet (22 weeks of age, $n=4$ ) (open squares) markedly abrogated MAP elevation to approximately that of non-Tg mice (filled squares) fed chow diet. ${ }^{*} P<0.05$ vs. Tg mice fed a high-salt diet.

switching from low to high salt intakes (51-54). The apparent activation of the RAS in aP2-HSD1 mice prompted us to assess potential salt sensitivity. MAP in 23-week-old non-Tg mice $(n=4)$ fed a high-salt $(8 \%)$ diet for 3 weeks did not significantly increase compared with initial values when fed a low-salt (1\%) chow diet, consistent with previous literature showing that FVB mice are salt-resistant (55) (Figure 2a, left). On the other hand, after 3 weeks on a high-salt diet, MAP in $\operatorname{Tg}$ mice $(n=5)$ was significantly elevated (by $10-20 \mathrm{mmHg}$ ) for most of the day compared with initial values $(P<0.05$, except 0700 hours to 0800 hours, 1500 hours to 1600 hours, and 1800 hours to 2400 hours) (Figure 2a, right). Notably, BP elevation was exaggerated, especially in the late dark (100 hours to 500 hours) and light phases (800 hours to 1300 hours) (Figure 2a, right). Thus, the daily profile of MAP in $\mathrm{Tg}$ mice after high salt loading appeared flatter with a higher nadir, reminiscent of "non-dipper" type salt-sensitive hypertension in humans, which is known to be closely associated with multiple metabolic and cardiovascular risks $(56,57)$.

Furthermore, 4 days of low-dose administration of GA0113 (0.1 mg/kg body weight) to Tg mice fed a highsalt diet markedly abrogated MAP elevation to approximately the levels of non-Tg mice fed a chow diet (Figure $2 \mathrm{~b}$ ), indicating that the salt-sensitive component in hypertension of Tg may largely be RAS-dependent.

Histological changes in kidneys of aP2-HSD1 mice. In 25 -week-old mice, there was no appreciable difference in the whole heart weight/body weight ratio $(\mathrm{mg} / \mathrm{g})$, an index of cardiac hypertrophy (58), between non- $\operatorname{Tg}(5.8 \pm 0.4, n=8)$ and $\operatorname{Tg}(6.0 \pm 0.5$, $n=11)$ mice. The difference in kidney weight between non- $\mathrm{Tg}(246 \pm 23 \mathrm{mg})$ and $\mathrm{Tg}(287 \pm 32 \mathrm{mg})$ mice also did not reach statistical significance.

Histological examination of the kidneys of aP2-HSD1 mice (males 24 weeks of age, $n=5$ ) showed evident dis- tal tubular hypertrophy and hyperplasia starting immediately proximal to the macula densa (Figure 3, a and $b$ ). The enlarged tubules in Tg mice (Figure $3 b$ ) measured up to 2.5 times the tubular cross-sectional area of non-Tg mice (Figure 3a). The enlarged tubular cells in Tg mice exhibited increased cytoplasmic granularity, most markedly in the basal cytoplasm, with apical nuclear displacement (Figure 3d), while none of these changes were seen in non-Tg mice (Figure 3c). Under electron microscopy, increased eosinophilic granularity corresponded to an increased number of mitochondria (data not shown). There was also a modest degree of hyperplasia of the juxtaglomerular apparatus in Tg mice (Figure 3f) but not in non-Tg mice (Figure 3e). In both genotypes, glomeruli, proximal tubules, medullary thick ascending limb, and blood vessels were histologically unaltered.

These findings in kidneys of $\mathrm{Tg}$ mice are consistent with chronic high BP, especially salt-sensitive or angiotensin II-mediated hypertension $(59,60)$. It is interesting to note that the features are similar although less marked than seen in the distal tubule of mice lacking $11 \beta$-HSD type 2 (11 $\beta$-HSD $2^{-/-}$mice), which demonstrate the major features of the syndrome of apparent mineralocorticoid excess (SAME) (39). $11 \beta$-HSD2 is a potent dehydrogenase that inactivates glucocorticoid in cells $(19,20)$. Thus, it is possible that elevated levels of aldosterone in $\mathrm{Tg}$ mice drive mineralocorticoid receptor activation and may be responsible, at least in part, for the distal tubular pathology in Tg mice. Moreover, a recent study has demonstrated that $11 \beta-H S D 2^{-/-}$mice have pronounced endothelial dysfunction causing enhanced norepinephrine-mediated contraction (61). In this context, further studies addressing endothelial and vascular function are required to better understand the renal histological changes observed in aP2-HSD1 mice. 

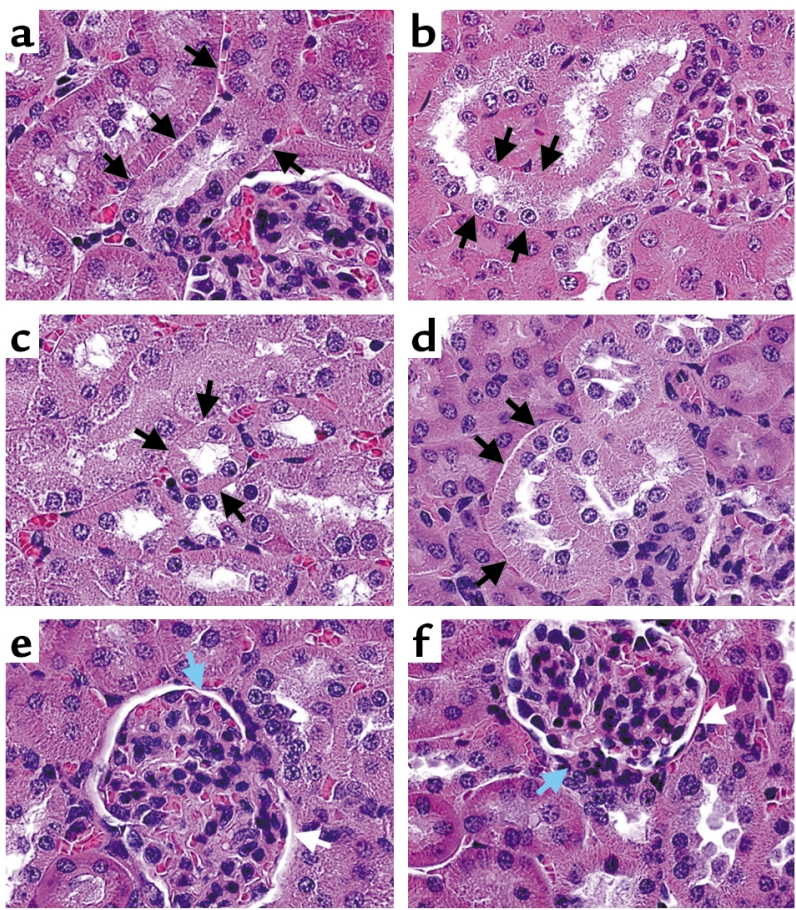

\section{Discussion}

The aP2-HSD1 mouse serves as a unique model to approach the molecular mechanism of bypertension in the metabolic syndrome. The present study demonstrates that aP2-HSD1 mice have hypertension accompanied by features of the metabolic syndrome, including visceral fat accumulation and insulin and leptin resistance. Our data suggest that an activated circulating RAS, along with hyperinsulinemia and hyperleptinemia, are responsible for hypertension in the aP2-HSD1 mouse. Previous studies have shown that RAS blockade is less effective in hypertensive patients with low renin activity $(45,51,54)$. On the other hand, systemic RAS activation may be responsible, at least in part, for the excellent effectiveness of the AT-1 receptor antagonist on hypertension of aP2-HSD1 mice. In this context, further studies are necessary to elucidate whether activated RAS is involved in the pathogenesis of human hypertension in the metabolic syndrome, as it is in aP2-HSD1 mice.

Although naturally occurring rodent models resembling human metabolic syndrome such as Zucker diabetic fatty (ZDF) (62) and obese spontaneous hypertensive (SHROB) rats (63) have been characterized, the pathophysiologic relationship between hypertension and the metabolic syndrome in these models remains incompletely understood. Moreover, it is important to note that diet-induced obese models, even accompanied by hyperleptinemia and hyperinsulinemia, do not necessarily have hypertension $(64,65)$. In this context, aP2HSD1 mice are a unique experimental model for studying the pathophysiology of hypertension in a close analogue of the human metabolic syndrome (Figure 4).

Both animal model studies and clinical investigations have demonstrated that pharmacologic blockade of the

\section{Figure 3}

Histological examination of the kidneys of aP2-HSD1 mice and non$\mathrm{Tg}$ littermates (24 weeks of age, male, $n=5$ in each group). All are $\mathrm{H} \& \mathrm{E}$ stained sections photographed at an original magnification of $\times 400$. (a and $\mathbf{b}$ ) Distal tubular hyperplasia and hypertrophy with an origin immediately proximal to the macula densa are seen in Tg mice (b) compared with non-Tg littermates (a). The enlarged tubules in $\mathrm{Tg}$ mice (b, arrows) measured up to 2.5 times the cross-sectional area of tubules in non-Tg mice (a, arrows). (c and d) Distal tubular hypertrophy with increased cytoplasmic granularity and apical nuclear displacement are seen in Tg mice (d, arrows) compared with the normal, smaller distal tubules in non-Tg mice (c, arrows). (e and $\mathbf{f}$ ) Moderate hyperplasia of the juxtaglomerular apparatus (JGA) at the vascular pole of the glomerulus is found in $\mathrm{Tg}$ mice ( $\mathbf{f}$ ) compared with non- $\mathrm{Tg}$ mice (e). White and blue arrows indicate glomeruli and JGAs, respectively. In both genotypes, glomeruli, proximal tubules, medullary thick ascending limb, and blood vessels were histologically unaltered.

RAS by angiotensin I-converting enzyme (ACE) inhibitors and AT- 1 receptor antagonists can ameliorate whole-body insulin resistance (66-68). It has been shown that angiotensin II inhibits insulin-stimulated PI3K activity by interfering with the docking of insulin substrate-1 (IRS-1) with the p85 regulatory subunit of PI3K (69). A recent in vivo study suggested that impaired insulin signaling located downstream from PI3K activation is involved in angiotensin II-induced insulin resistance (70). Collectively, overactivity of the RAS is likely to contribute to insulin resistance in hypertensive individuals with metabolic complications. Consistent with this notion, after only 5 days' administration of an AT-1 receptor antagonist, GA0113 $(0.1 \mathrm{mg} / \mathrm{kg}$ body weight $)$, the profile of insulin tolerance in 26-week-old aP2-HSD1 mice $(n=4)$ was significantly improved compared with that performed 3 days before the start of treatment.

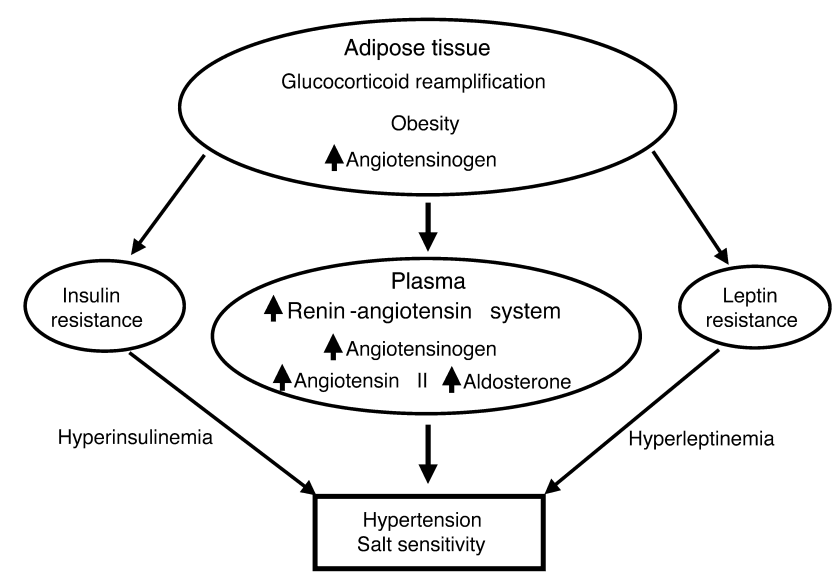

\section{Figure 4}

Hypothetical representation of the pathophysiology of hypertension in aP2-HSD1 mice. Along with causing hyperinsulinemia and hyperleptinemia, reamplification of glucocorticoid action in adipose tissue leads to the activation of the circulating RAS and results in blood pressure elevation. This mouse model may provide a unique experimental system to better understand the pathophysiology of hypertension in human metabolic syndrome. 
Percentages of initial value of glucose were: at 30 minutes, $72 \% \pm 3 \%$ basal versus $59 \% \pm 6 \%$ treated, $P<0.03$; 60 minutes, $61 \% \pm 4 \%$ basal versus $48 \% \pm 3 \%$ treated, $P<0.02 ; 90$ minutes, $66 \% \pm 3 \%$ basal versus $44 \% \pm 6 \%$ treated, $P<0.03$. Serial values in non-Tg mice $(n=6)$ treated with saline were $48 \% \pm 3 \%$ at 30 minutes, $30 \% \pm 4 \%$ at 60 minutes, and $34 \% \pm 2 \%$ at 90 minutes, respectively. These data suggest that circulating RAS activation may contribute to insulin resistance in aP2-HSD1 mice. Conversely, it is tempting to speculate that improved insulin sensitivity caused by RAS blockade may also contribute to the BP drop in Tg mice.

In summary, the present study demonstrates that aP2-HSD1 mice are hypertensive with apparent activation of the circulating RAS and features of the metabolic syndrome. The data highlight the potential role of adipose glucocorticoid reactivation in the pathophysiology of hypertension seen in the metabolic syndrome. The aP2-HSD1 mouse provides a unique experimental model in which to better understand the molecular mechanism of hypertension in the metabolic syndrome. These findings also suggest that drugs that inhibit $11 \beta$-HSD 1 in adipose tissue would improve the syndrome's myriad pathological features, including hypertension.

\section{Acknowledgments}

We thank J.J. Morton for angiotensin II assays and T. Collidge for help in histological preparation. This work was supported by grants from NIH and Eli Lilly and Co. (to J.S. Flier), a program grant from the Wellcome Trust (J.R. Seckl and J.J. Mullins), grants from NIH NIDDK (DK-59751 and DK-56116 to J.K. Elmquist), and from the Transgenic Core Facility of the Boston Obesity Nutrition Research Center and the Animal Physiology Core of the Diabetes, Endocrinology Research Center (to J.S. Flier). H. Masuzaki held research fellowships from Uehara Memorial Foundation, Welfide Medicinal Research Foundation, and Yamanouchi Foundation for Research on Metabolic Disorders (Tokyo, Japan). We are grateful to $\mathrm{T}$. Williams, M. Overton, Y. Minokoshi, B.B. Kahn, J.K. Hamm, E.E. Kershaw, S. Blueher, L. Oksanen, S. Hileman, R. Rossi, T. Hampton, E. Rosen, Y.-B. Kim, H. Fang, M. Kokoeva, and P. Kievit for helpful discussions.

1. Spiegelman, B.M., and Flier, J.S. 2001. Obesity and the regulation of energy balance. Cell. 104:531-543.

2. Matsuzawa, Y., Funahashi, T., and Nakamura, T. 1999. Molecular mechanism of metabolic syndrome $\mathrm{X}$ : contributions of adipocytokines, adipocyte-derived bioactive substances. Ann. N. Y. Acad. Sci. 892:146-154.

3. Kahn, B.B., and Flier, J.S. 2000. Obesity and insulin resistance. J. Clin. Invest. 106:473-481.

4. Aizawa-Abe, M., et al. 2000. Pathophysiological role of leptin in obesityrelated hypertension. J. Clin. Invest. 105:1243-1252.

5. Busetto, L. 2001. Visceral obesity and the metabolic syndrome. Effect of weight loss. Nutr. Metab. Cardiovasc. Dis. 11:195-204.

6. Kopelman, P.G. 2000. Obesity as a medical problem. Nature. 404:635-643.

7. Diep, Q.N., et al. 2002. Structure, endothelial function, cell growth, and inflammation in blood vessels of angiotensin II-induced rats. Role of peroxisome proliferator-activated receptor- $\gamma$. Circulation. 105:2296-2302.

8. Hodge, G., Ye, V.Z., and Duggan, K.A. 2002. Salt-sensitive hypertension resulting from nitric oxide synthase inhibition is associated with loss of regulation of angiotensin II in the rat. Exp. Physiol. 87:1-8.

9. Wajchenberg, B.L. 2000. Subcutaneous and visceral adipose tissue: their relation to the metabolic syndrome. Endocr. Rev. 21:697-738.
10. Inagami, T., et al. 1999. Angiotensin receptors: molecular biology and signalling. Clin. Exp. Pharmacol. Physiol. 26:544-549.

11. Walker, W.G., Whelton, P.K., Saito, H., Russel, R.P., and Hermann, J. 1979. Relation between blood pressure and renin, renin substrate, angiotensin II, aldosterone and urinary sodium and potasium in 574 ambulatory subjects. Hypertension. 1:287-291.

12. Lalouel, J.M., Rohrwasser, A., Terreros, D., Morgan, T., and Ward, K. 2001. Angiotensinogen in essential hypertension: from genetics to nephrology. J. Am. Soc. Nephrol. 12:606-615.

13. Ailhaud, G., Teboul, M., and Massiera, F. 2002. Angiotensinogen, adipocyte differentiation and fat mass enlargement. Curr. Opin. Clin. Nutr. Metab. 5:385-389.

14. Rotimi, C., et al. 1997. Hypertension, serum angiotensinogen, and molecular variants of the angiotensinogen gene among Nigerians. Circulation. 95:2348-2350.

15. Pratt, J.H., et al. 1998. Serum angiotensinogen concentration in relation to gonadal hormones, body size, and genotype in growing young people. Hypertension. 32:875-879.

16. Massiera, F., et al. 2001. Adipose angiotensinogen is involved in adipose tissue growth and blood pressure regulation. FASEB J. 15:2727-2739.

17. Montague, C.T., and O'Rahilly, S. 2000. The perils of portliness. Cause and consequences of visceral adipocity. Diabetes. 49:883-888.

18. Peeke, P.M., and Chrousos, G.P. 1995. Hypercortisolism and obesity. Ann. N. Y. Acad. Sci. 771:665-676.

19. Seckl, J.R., and Walker, B.R. 2001. Minireview: 11ß-hydroxysteroid dehydrogenase type 1 - a tissue-specific amplifier of glucocorticoid action. Endocrinology. 142:1371-1376.

20. Sandeep, T.C., and Walker, B.R. 2001. Pathophysiology of modulation of local glucocorticoid levels by 11 beta-hydroxysteroid dehydrogenases. Trends Endocrinol. Metab. 12:446-453.

21. Tomlinson, J.W., et al. 2002. Absence of Cushingoid phenotype in a patient with Cushing's disease due to defective cortisone to cortisol conversion. J. Clin. Endocrinol. Metab. 87:57-62.

22. Bjorntorp, P., and Rosmond, R. 2000. Obesity and cortisol. Nutrition. 16:924-936.

23. Chrousos, G.P., and Gold, P.W. 1992. The concept of stress and stress system disorders. Overview of physical and behavioral homeostasis. JAMA. 267:1244-1252.

24. Reynolds, R.M., et al. 2002. Skeletal muscle glucocorticoid receptor density and insulin resistance. JAMA. 287:2505-2506.

25. Rask, E., et al. 2001. Tissue-specific dysregulation of cortisol metabolism in human obesity. J. Clin. Endocrinol. Metab. 86:1418-1421.

26. Rask, E., et al. 2002. Tissue-specific changes in peripheral cortisol metabolism in obese women: increased adipose $11 \beta$-hydroxysteroid dehydrogenase type 1 activity. J. Clin. Endocrinol. Metab. 87:3330-3336.

27. Whorwood, C.B., Donovan, S.J., Flanagan, D., Phillips, D.I.W., and Byrne, C.D. 2002. Increased glucocorticoid receptor expression in human skeletal muscle cells may contribute to the pathogenesis of the metabolic syndrome. Diabetes. 51:1066-1075.

28. Paulmyer-Lacroix, O., Bouliu, S., Oliver, C., Alessi, M.-C., and Grino, M. 2002. Expression of the mRNA coding for $11 \beta$-hydroxysteroid dehydrogenase type 1 in adipose tissue from obese patients: an in situ hybridization study. J. Clin. Endocrinol. Metab. 87:2701-2705.

29. Livingstone, D.E., et al. 2000. Understanding the role of glucocorticoids in obesity: tissue-specific alterations of corticosterone metabolism in obese Zucker rats. Endocrinology. 141:560-563.

30. Livingstone, D.E., Kenyon, C.J., and Walker, B.R. 2000. Mechanisms of dysregulation of 11beta-hydroxysteroid dehydrogenase type 1 in obese Zucker rats. J. Endocrinol. 167:533-539.

31. Kotelevstev, Y., et al. 1997. 11beta-hydroxysteroid dehydrogenase type 1 knockout mice show attenuated glucocorticoid-inducible responses and resist hyperglycemia on obesity or stress. Proc. Natl. Acad. Sci. U. S. A. 94:14924-14929.

32. Morton, N.M., et al. 2001. Improved lipid and lipoprotein profile, hapatic insulin sensitivity, and glucose tolerance in $11 \beta$-hydroxysteroid dehydrogenase type 1 null mice. J. Biol. Chem. 276:41293-41300.

33. Morton, N.M., et al. 2002. 11ß-hydroxysteroid dehydrogenase type 1 null mice resist dietary obesity and its metabolic consequences. Program of the 84th annual meeting of the Endocrine Society. San Francisco, California, USA. 87 (Abstr.)

34. Masuzaki, H., et al. 2001. A transgenic model of visceral obesity and the metabolic syndrome. Science. 294:2166-2170.

35. Yamamoto, H., et al. 2002. Glucagon-like peptide-1 receptor stimulation increases blood pressure and heart rate and activates autonomic regulatory neurons. J. Clin. Invest. 110:43-52.

36. Williams, T.D., Chambers, J.B., Henderson, R.P., Rashotte, M.E., and Overton, J.M. 2002. Cardiovascular responses to caloric restriction and thermoneutrality in C57BL/6J mice. Am. J. Physiol. Regul. Integr. Comp. Physiol. 282:R1459-R1467.

37. Ebisu, H., et al. 1999. Pharmacologic profiles of GA0113, a novel quinoline derivative angiotensin II $\mathrm{AT}_{1}$-receptor antagonist. J. Cardiovasc. Pharmacol. 34:526-532. 
38. Morton, J.J., and Wallace, E.C.H. 1983. The importance of the reninangiotensin system in the development and maintenance of hypertension in the two-kidney one clip hypertensive rat. Clin. Sci. 64:359-370.

39. Kotelevtsev, Y., et al. 1999. Hypertension in mice lacking $11 \beta$-hydroxysteroid dehydrogenase type 2. J. Clin. Invest. 103:683-689.

40. Llorens-Cortes, C., Greenberg, B., Huang, H., and Corval, P. 1994. Tissue expression and regulation of type 1 angiotensin II receptor subtypes by quantitative reverse transcriptase chain reaction analysis. Hypertension. 24:538-548.

41. Basett, M.H., Zhang, Y., Clyne, C., White, P.C. and Rainey, W.E. 2002. Differential regulation of aldosterone synthetase and 11beta-hydroxylase transcription by steroidogenic factor-1. J. Mol. Endocrinol. 28:125-135.

42. Butz, G.M., and Davisson, R.L. 2001. Long-term telemetric measurement of cardiovascular parameters in awake mice: a physiological genomics tool. Physiol. Genomics. 5:89-97.

43. Shimada, K., et al. 2001. Early morning surge in blood pressure. Blood Press. Monit. 6:349-353.

44. Redon, J., Rosa-Cusachs, A., and Mora-Macia, J. 2002. Uncontrolled early morning blood pressure in medicated patients: the ACAMPA study. Blood Press. Monit. 7:111-116.

45. Engeli, S., and Sharma, A.M. 2002. Emerging concepts in the pathophysiology and treatment of obesity-associated hypertension. Curr. Opin. Cardiol. 17:355-359.

46. Frederich, R.C., Jr., Kahn, B.B., Peach, M.J., and Flier, J.S. 1992. Tissue-specific nutritional regulation of angiotensinogen in adipose tissue. Hypertension. 19:339-344.

47. Aubert, J., Darimont, C., Safonova, I., Ailhoud, G., and Negrel, R. 1997. Regulation by glucocorticoids of angiotensinogen gene expression and secretion in adipose cells. Biochem. J. 328:701-706.

48. van Harmelen, V., et al. 2000. Increased adipose angiotensinogen gene expression in human obesity. Obes. Res. 8:337-341.

49. van Harmelen, V., et al. 2000. The association of human adipose angiotensinogen gene expression with abdominal fat distribution in obesity. Int. J. Obes. Relat. Metab. Disord. 24:673-678.

50. Miller, J.Z., Weinberger, M.H., Christian, J.C., and Daugherty, S.A. 1987. Familial resemblance in the blood pressure response to sodium retention. Am. J. Epidemiol. 126:822-830.

51. Poch, E., et al. 2001. Molecular basis of salt sensitivity in human hypertension. Evaluation of renin-angiotensin-aldosterone system gene polymorphisms. Hypertension. 38:1204-1209.

52. Cowley, A.W., Jr. 1997. Genetic and nongenetic determinants of salt sensitivity and blood pressure. Am. J. Clin. Nutr. 65(Suppl.):S587-S593.

53. Weinburger, M.H., Miller, J.Z., Luft, F.C., Grim, C.E., and Finberg, N.S. 1986. Definitions and characteristics of sodium sensitivity and blood pressure resistance. Hypertension. 8(Suppl. II):II127-II134.
54. De la Sieera, A., et al. 1996. Fluid, ionic and hormonal changes induced by high salt intake in salt-sensitive and salt-resistant hypertensive patients. Clin. Sci. 91:155-161.

55. Sugiyama, F., et al. 2001. Concordance of murine quantitative trait loci for salt-induced hypertension with rat and human loci. Genomics. 71:70-77.

56. O'Brien, E., Sheridan, J., and O'Malley, K. 1988. Dippers and non-dippers. Lancet. 13:397.

57. Engeli, S., and Sharma, A.M. 2001. The renin-angiotensin system and natriuretic peptides in obesity-associated hypertension. J. Mol. Med. 79:21-29.

58. Masuzaki, H., et al. 1996. Regulation of very-low-density lipoprotein receptor in hypertrophic rat heart. Circ. Res. 78:8-14.

59. Johnson, R.J., et al. 1992. Renal injury from angiotensin II-mediated hypertension. Hypertension. 19:464-474.

60. Pradevand, S., et al. 1999. A mouse model for Liddle's syndrome. J. Am. Soc. Nephrol. 10:2527-2533.

61. Hadoke, P.W., et al. 2001. Endothelial cell dysfunction in mice after transgenic knockout of type 2, but not type 1, 11beta-hydroxysteroid dehydrogenase. Circulation. 104:2832-2837.

62. Stepp, D.W., and Frisbee, J.C. 2002. Augmented adrenergic vasoconstriction in hypertensive diabetic obese Zucker rats. Am. J. Physiol. Heart Circ. Physiol. 282:H816-H820.

63. Villiquette, R.A., Koletsky, R.J., and Ernsberger, P. 2002. Plasma glucagon and free fatty acid responses to a glucose load in the obese spontaneous hypertensive rat (SHROB) model of metabolic syndrome X. Exp. Biol. Med. 227:164-170.

64. Mills, E., Kuhn, C.M., Feiglos, M.N., and Surwit, R. 1993. Hypertension in CB57BL/6J mouse model of non-insulin dependent diabetes mellitus. Am. J. Physiol. 264:R73-R78.

65. Contreras, R.J., and Williams, V.L. 1989. Dietary obesity and weight cycling: effects on blood pressure and heart rate in rats. Am. J. Physiol. 256:R1209-R1219.

66. Katovich, M.J., et al. 2001. Gene therapy attenuates the elevated blood pressure and glucose intolerance in an insulin-resistant model of hypertension. J. Hypertens. 19:1553-1558.

67. Montani, J.P., Antic, V., Yang, Z., and Dulloo, A. 2002. Pathways from obesity to hypertension: from the perspective of a vicious triangle. Int. J. Obes. Relat. Metab. Disord. 26(Suppl. 2):S28-S38.

68. Henriksen, E.J., Jacob, S., Kinnick, T.R., Teachey, M.K. and Krekler, M. 2001. Selective angiotensin II receptor antagonism reduces insulin resistance in obese Zucker rats. Hypertension. 38:884-890.

69. Folli, F., et al. 1999. Crosstalk between insulin and angiotensin II signalling systems. Exp. Clin. Endocrinol. Diabetes. 107:133-139.

70. Ogihara, T., et al. 2002. Angiotensin II-induced insulin resistance is associated with enhanced insulin signaling. Hypertension. 40:872-879. 